\title{
Hyper Gravity-Induced Transients in Phycomyces as Measured by Single Beam Spectrophotometer on the Sounding Rocket TEXUS 50
}

\author{
Werner Schmidt ${ }^{1,2}$ \\ ${ }^{1}$ Fachbereich Biologie, Philipps-Universität Marburg, Marburg, Germany \\ ${ }^{2}$ Fachbereich Biologie, Universität Konstanz, Konstanz, Germany \\ Email: w.2.schmidt@gmx.de
}

How to cite this paper: Schmidt, W. (2018) Hyper Gravity-Induced Transients in Phycomyces as Measured by Single Beam Spectrophotometer on the Sounding Rocket TEXUS 50. Journal of Modern Physics, 9 , 273-286.

https://doi.org/10.4236/jmp.2018.92019

Received: November22, 2017

Accepted: January 21, 2018

Published: January 24, 2018

Copyright $\odot 2018$ by author and Scientific Research Publishing Inc. This work is licensed under the Creative Commons Attribution International License (CC BY 4.0).

http://creativecommons.org/licenses/by/4.0/ (c) (i) Open Access

\begin{abstract}
In the first paper of two referring to the TEXUS 50 campaign using micro dual wavelength spectrometers (MDWS) we kinetically determined the threshold ${ }^{1}$ for GIACs (gravity-induced absorption changes) in Phycomyces to be lower than $25 \times 10^{-3} \mathrm{~g}$ (http://file.scirp.org/pdf/JMP_2015082810060783.pdf). In this second paper, we attended measurement of GIAC-spectra. Unexpectedly, during the upwards movement, i.e. the hypergravity phase up to top acceleration values reaching $11.6 \mathrm{~g}$ at $35.4 \mathrm{~s}$ after liftoff we observed transient GIAC-spectra ranging from 380 to $750 \mathrm{~nm}$. In addition, during the whole acceleration phase of $68.2 \mathrm{~s}$, another component near $700 \mathrm{~nm}$ develops which remains stable during the whole "free fall trajectory parabola" for $381.3 \mathrm{~s}$. The subsequent reentry of the rocket leads to extraordinary deceleration values up 37.8 g, completely destroying Phycomyces sporangiophores excluding their spectral measurement. During the microgravity phase and by centrifuge operation we were unable to detect any GIAC-spectra (in contrast to kinetic MDWS-measurements, first paper).
\end{abstract}

\section{Keywords}

MDWS (Micro-Dual Wavelength Spectrophotometer), Single Beam Spectrophotometer (SBS), Diode Array Spectrometer (USB-2000+, Ocean Optics), GIAC (Gravity-Induced Absorption Change), Phycomyces blakesleeanus, Sporangiophore, Micro- and Hypergravity, Texus 50, Sounding Rocket, Graviperception. Hyper Gravity-Induced Transients

${ }^{1}$ The "threshold" is the smallest stimulus where a response is observed. It depends on the sensitivity of the measuring set-up. 


\section{Introduction}

The Micro Dual Wavelength Spectrometer (MDWS) only allows measurements at individual wavelengths of the spectrum defined by selected light emitting diodes (LEDs) [1]. It does not allow to measure complete spectra with a comparable high sensitivity. Therefore, we attempted a complementary measurement of the complete spectrum of GIACs in Phycomyces sporangiophores (SPPHs, Figure 1), during the start phase of hypergravity and the subsequent phase of microgravity using a single beam spectrophotometer (diode array spectrophotometer SBS, USB-2000+ by Ocean Optics). However, due to its fundamentally much lower sensitivity of the SBS compared to the MDWS we were not able to detect any GIACs on a spectral basis during the microgravity phase. During our first evaluation of the TEXUS 50 data [1] we only focussed on the originally envisaged data obtained during microgravity, ignoring data obtained during the start phase, i.e. hypergravity. Surprisingly, just during the phase of strong hypergravity ( 0 to $37 \mathrm{~s}$, acceleration up to $11.6 \mathrm{~g}$ ) we observed pronounced and intermediate GIAC-spectra. In addition, another but smaller peak comes up during the late state of hypergravity, further standing in the subsequent microgravity phase. These GIACs generated under hypergravity conditions appear to be of biological rather than artificial background.

\section{Material and Methods}

\subsection{Sounding Rocket TEXUS 50}

We participated in the $50^{\text {th }}$ jubilee sounding rocket campaign:

(http://www.spacedaily.com/reports/Swedish_Space_Corporation_Celebrates_50 th_Anniversary_of_Esrange_Space_Center_999.html). The Texus 50 was started on the $12^{\text {th }}$ of April 2013, burn out of the first rocket motor after $11.6 \mathrm{~s}$ at a height of about $8 \mathrm{~km}$, burn out of the second rocket motor after $43.9 \mathrm{~s}$ at a height of about $100 \mathrm{~km}$ reaching a velocity of app. $10100 \mathrm{~km} \mathrm{~h}^{-1}$. After "YoYo-despin"
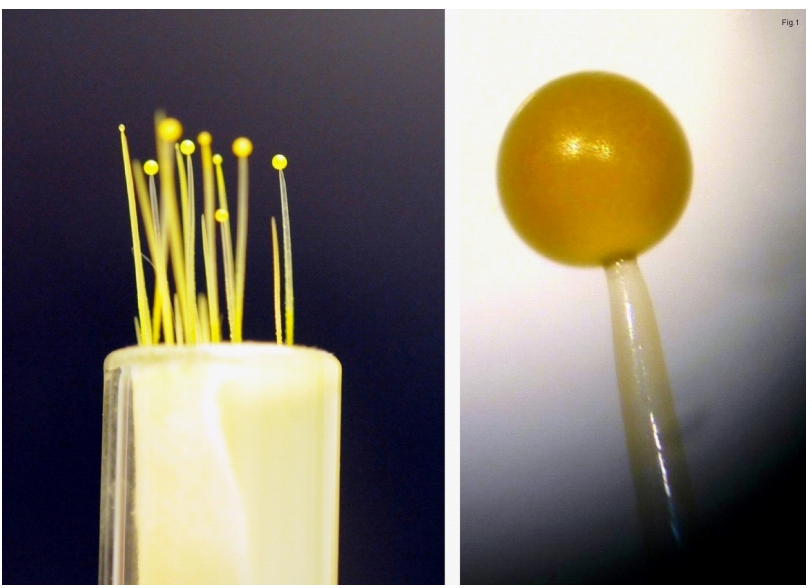

Figure 1. Left: Photograph of a typical arrangement of 10 to 15 sporangiophores (SPPHs) of Phycomyces in a glass vial as used for the experiments. Right. Microscopic picture of a single Sporangium. 
the rocket "falls" in a fixed direction through a narrow parabola (cf. Figure 1 in [1]) and is completely rotational-free as tested by a three-dimensional on-board magnetometer. The greatest height of $261.2 \mathrm{~km}$ is reached after $261.9 \mathrm{~s}$. During the whole phase of microgravity $\left(<10^{-5} \mathrm{~g}\right.$, between 68.2 to $449.5 \mathrm{~s}$ after liftoff) the payload remains completely stable in space allowing the fine-tuned application of gravitational forces by the rotary platform, particularly during the subsequent downwards movement. Gravitational ramps of 5, 25, 50, 75 and $100 \mathrm{mg}$ were applied, giving rise to the accompanying GIACs as measured with the MDWS [1].

\subsection{The Two Applied Concepts of Optical Spectroscopy}

The Micro Dual Wavelength Spectrometer (MDWS) has been developed by us and described previously in more detail [2] [3]. The MDWS is capable of measuring extremely small optical absorption/reflection changes $\left(<10^{-5} \mathrm{~A}\right)$ and is at least 1000 times more sensitive(!) than common double and single beam spectrophotometers, depending on the measuring conditions [4]. More details are given in the first paper [1].

The Single Beam Spectrophotometer (SBS): In contrast to classical double beam spectroscopy the SBS allows a much simplified set-up and extensive usage of special computer software. In order to measure GIAC-spectra in this present $2^{\text {nd }}$ paper of this series of sounding rocket (TEXUS 50) experiments we used the miniature SBS as based on a diode array technology (USB-2000+, Ocean Optics, Figure 2). It is highly flexible, small, sensitive (dynamic range $8.5 \times 10^{8}$ for single acquisition), and fast: full scan from $3 \mathrm{~ms}$ up to $0.283 \mathrm{~s}$ as in the present work, depending on used conditions such as averaging, boxcar width, time delay and slit width. Using two sets of white and blue LEDs each for sufficient illumination of the SPPHs we monitored the whole spectrum from 350 to $875 \mathrm{~nm}$ within 0.283 s. The program was written by Astrium/Bremen. In order to increase illumination strength and thereby sensitivity, no entrance slit at all was used, only the light fiber diameter of $400 \mu \mathrm{m}$ serving as wavelength limiter. As in the first paper [1], because of the structure of SPPHs and for technical reasons we measured

$(89.1 \times 63.3 \times 34.4)$

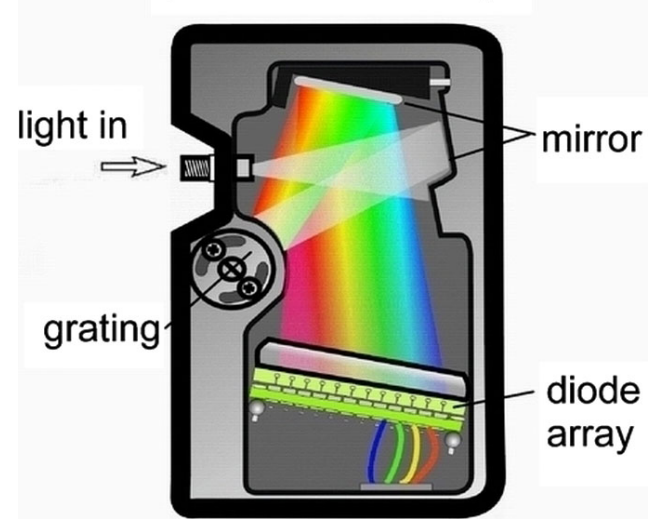

Figure 2. Sketch and light pass of the diode array spectrophotometer USB-2000+ (Ocean Optics). 
reflectance rather than absorption as suggested by its name. Important to note generally: when measuring very small optical signals in various SBS-modes such as absorption, fluorescence or reflection the absolute measure is inevitably lost (e.g. dark currents, light scattering, see [4]). Thus, the baseline has to be defined manually (cf. Figure 4, Figure 5, Figure 7, Figure 10).

Nevertheless, due to the relative small amount of reflected light reaching the entrance of the spectrometer only a poor $\mathrm{SNR}^{3}$ is attained, anyway. However, after extensive averaging and by $\mathrm{FFT}^{2}$-smoothing using a suitable kernel for the integral, valuable information can be extracted even from noisy signals.

Figure 3 shows the 3D-plot of all four spectrophotometers embedded in the TEXUS 50 rocket (flight implementation plan by Astrium, MDWS, SBS; module TEM 06-33). A fixed platform is located in the upper part of the module, the rotary platform in the middle part. A 3D-magnetometer (not seen in this plot) is localized just below the fixed and on top the rotary platform (playing the essential role in the $3^{\text {rd }}$ forthcoming paper of this series). The bottom part contains the electronics, batteries and the motor drive of the rotary platform.

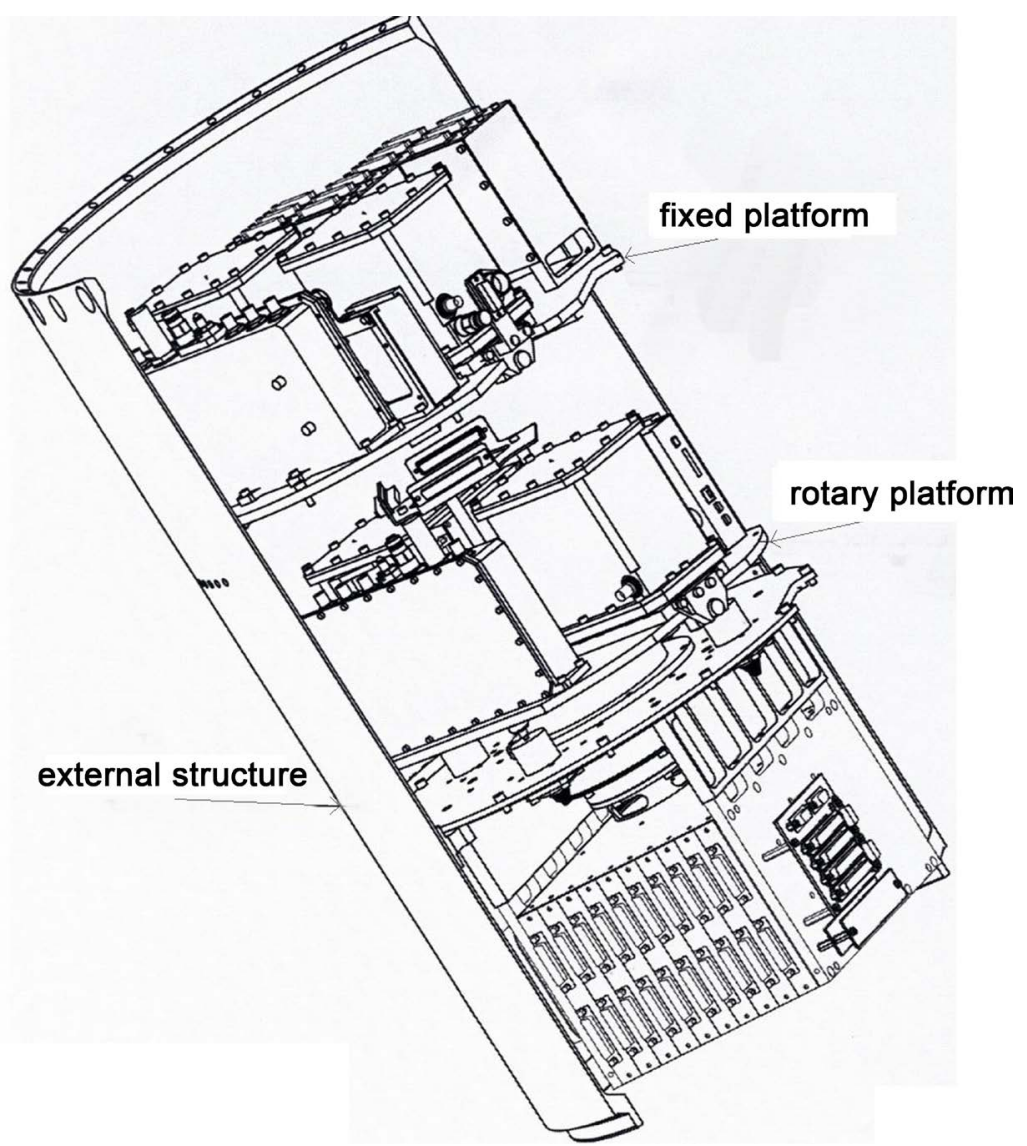

Figure 3. Three dimensional drawing of the whole module TEM 06-33 located in the lower part of the TEXUS 50 rocket (cf. Figure 1 in [1]). The upper part contains a fixed platform for one SBS and one MDWS. The middle part represents the centrifuge/rotating platform for one SBS and one MDWS, generating gravitational forces of 5, 25, 50, 75 and $100 \mathrm{mg}$ according to the ramps (Figure 5 in [1]). A 3D-magnetometer is placed just below the fixed and on top the rotary. The lower part is occupied by various electronic and radio elements. 


\subsection{Strains and Culture Conditions}

The left side of Figure 1 shows a hedge of SPPHs of Phycomyces blakesleeanus (Burgeff), NRRL1555 (-) originally obtained from the Northern Regional Research Laboratory, USDA, Peoria, IL, USA. Sporangiophores of Phycomyces were raised as described previously [5]. Right: Microscopic picture of a single sporangium. Highest geo- (and photo-) sensitivity is just below the spherical sporangium as shown. For short, they were grown in glass shell vials $(1 \mathrm{~cm}$ diameter $\times 4 \mathrm{~cm}$ height; Flachbodengläser, AR Klarglas, Münnerstädter Glaswarenfabrik, Münnerstadt, Germany) on a synthetic solid medium with glucose. Until the appearance of stage- $4 \mathrm{~b}$ sporangiophores (i.e. with sporangium) of 2.5 $\mathrm{cm}$ length the material was kept in transparent plastic boxes at ambient temperature $\left(19^{\circ} \mathrm{C}-21^{\circ} \mathrm{C}\right)$ under white incandescent light (fluence rate $\left.0.5 \mathrm{Wm}^{-2}\right)$.

\subsection{Telemetry}

The on board experiment is completely controlled and monitored from the base station via radio signals (TCE64-Telecommand-Encoder for 64 digital signals). These include various parameters such as currents of LEDs, averaging, boxcar, integration time, strobe frequency and correction for dark current. Common module data such as GPS, battery voltage, currents, temperatures, and amplification are monitored at $5 \mathrm{~Hz}$. GIAC data are monitored with 16 bit resolution at $500 \mathrm{~Hz}$ and completely stored in the on board micro-PC. For reasons of velocity only $1 / 16$ of all GIAC-data is transferred to the ground station via a KayserThrede-Module for numerical and graphical display on ground. In addition to GIAC-recording, this allows the baseline correction by the experimenter on-line. These control functions are performed on ground using a handheld console with all these functions available-in addition to a monitoring screen.

\section{Results and Discussion}

We measured 1281 (uncorrected) individual reflection spectra ranging from 350 to $850 \mathrm{~nm}$ during the start-(hypergravity, 0 to $68.2 \mathrm{~s}$ ) and the subsequent $\mathrm{mi}$ crogravity phase $\left(<10^{-5} \mathrm{~g}\right.$ between $68.2 \mathrm{~s}$ to $\left.449.5 \mathrm{~s}\right)$ as shown in Figure 4. Thus, 3.36 spectra per second were monitored. The observed changes are large and the spectra are from the beginning of microgravity "compressed" to a single spectrum. In addition, $15 \mathrm{~s}$ after the liftoff another group of "densified" spectra is observed. This is explained by the (uncorrected) kinetical GIAC-signals for various wavelengths indicated as obtained by the SBS. Figure 5(b) shows the original g-course of all three components $\mathrm{x}, \mathrm{y}, \mathrm{z}$ as provided by the rocket companies (Kayser-Threde, Astrium). The G-scale is marked in readable characters (too small in the original graph), for the purpose of comparison the time scales of Figure 5(a) and Figure 5(b) are properly adjusted. The upper trace in Figure 5 (b) represents the main g-value in flight direction $\left(G_{z}\right)$.

Just after liftoff the $G_{z}$ signal decreases for $3 \mathrm{~s}$ from 7 to $3.8 \mathrm{~g}$ (rocket is set to rotation) to increase again to $6 \mathrm{~g}$, when the first rocket motor is separated after 


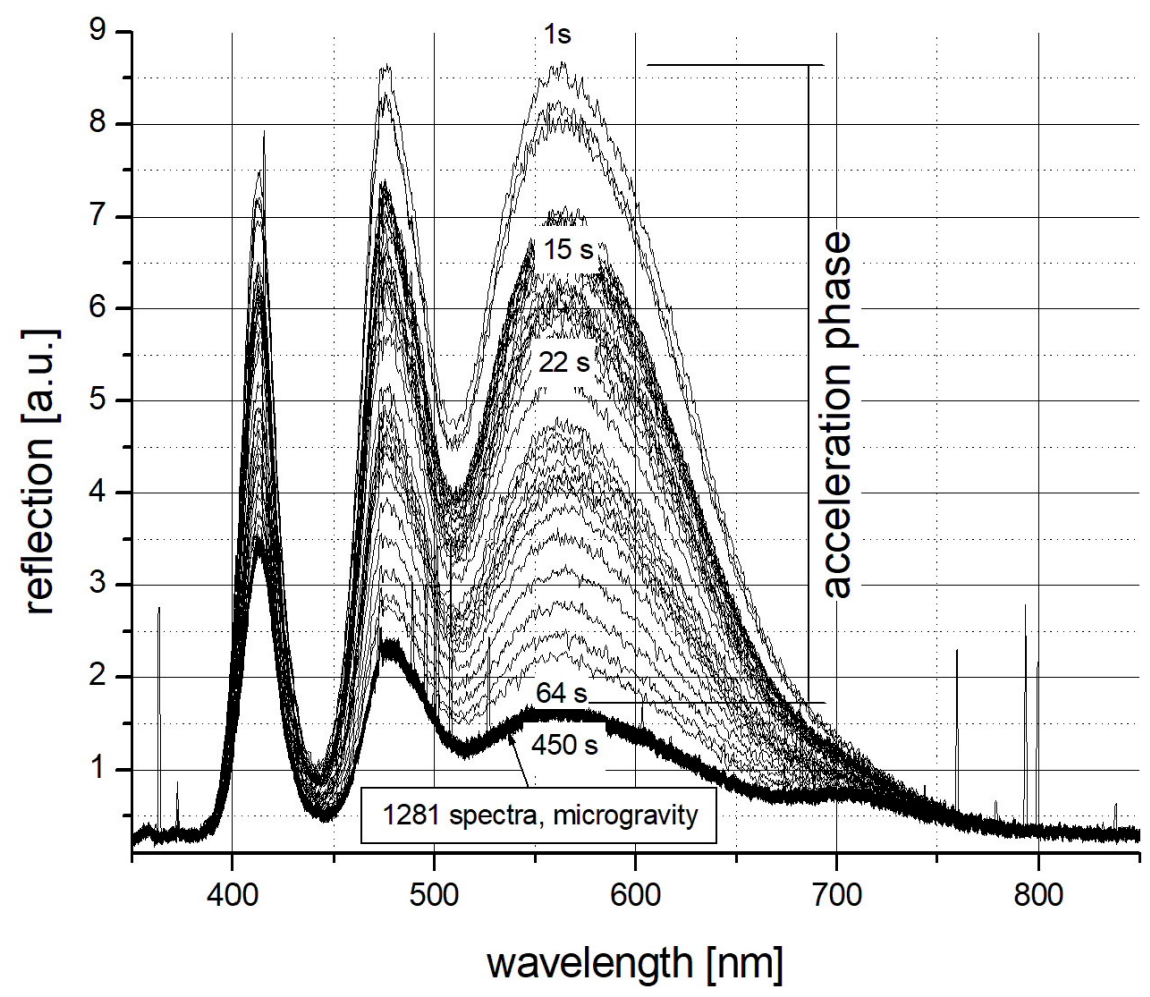

Figure 4. 1281 uncorrected reflection spectra ranging from 350 to $850 \mathrm{~nm}$ during the start (acceleration-phase, $0 . \mathrm{s}$ to $68.2 \mathrm{~s}$, hypergravity) and succeeding microgravity phase $\left(<10^{-5} \mathrm{~g}\right)$ between $68.2 \mathrm{~s}$ and $449.5 \mathrm{~s}$. Monitored were 3.36 spectra per second. The spectral and intensity changes during the start-, i.e. acceleration or hypergravity phase (max. $11.6 \mathrm{~g},>2 \mathrm{~g}$ by rockets rotation) are large and the spectra are from the beginning of microgravity "compressed" to a single spectrum. In addition, $15 \mathrm{~s}$ after liftoff there comes up another group of compressed spectra, which is explained below.

$12 \mathrm{~s}$ at a height about $8 \mathrm{~km}$. Then, for $3 \mathrm{~s} \mathrm{G}_{\mathrm{z}}$ is $-1 \mathrm{~g}$ (ground situation) and subsequently the rocket is boosted by the second motor up to a height approx. 100 $\mathrm{km}$ experiencing a maximum of $11.6 \mathrm{~g}$ after $35.5 \mathrm{~s}$ and a velocity of $10,100 \mathrm{~km} / \mathrm{h}$. Concluding, the course of the hypergravity-induced GIAC (Figure 5(a)) does not at all reflect the directly measured gravity course (Figure 5(b)). This is taken to indicate a biological intermediate rather than a (trivial) artifact which is further supported by the corrected GIAC-spectra and the MDWS signal as follows.

Figure 6 depicts 5 corrected GIAC-spectra $\left(\Delta \mathrm{R}=\log \mathrm{R}_{\mathrm{x}} / \mathrm{R}_{0}=\Delta \mathrm{A}\right)$ as selected and calculated from spectra in Figure 4 during hypergravity. $\mathrm{R}_{0}$ describes the (constant) reference spectrum after $68 \mathrm{~s}, \mathrm{R}_{\mathrm{x}}$ the various spectra at hypergravity at times $\mathrm{x}$ after liftoff. Clearly, during linear and rotary acceleration, smaller peaks shows up at 410 and $470 \mathrm{~nm}$, and a larger and broader one at $575 \mathrm{~nm}$. Comparison with the known absorption spectrum of Phycomyces sporangiophores (not shown here) does not allow to identify these peaks. However, during the $9^{\text {th }}$ DLR parabolic flight campaign some 11 years ago we measured the first GIAC-spectrum of wildtype Phycomyces blakesleeanus SPPHs by a novel SBS [7]. After extensive averaging and a subsequent fit by a higher polynom of $6^{\text {th }}$ order we obtained the smoothed difference spectrum $(1.8 \mathrm{~g}-0 \mathrm{~g})$ shown as dotted line in Figure 6 
("action spectrum"). This action spectrum obtained by a moderate hypergravity of $1.8 \mathrm{~g}$ within a minutes time scale appears to be largely exceled by hypergravity up to $11 \mathrm{~g}$ in the present case within seconds.
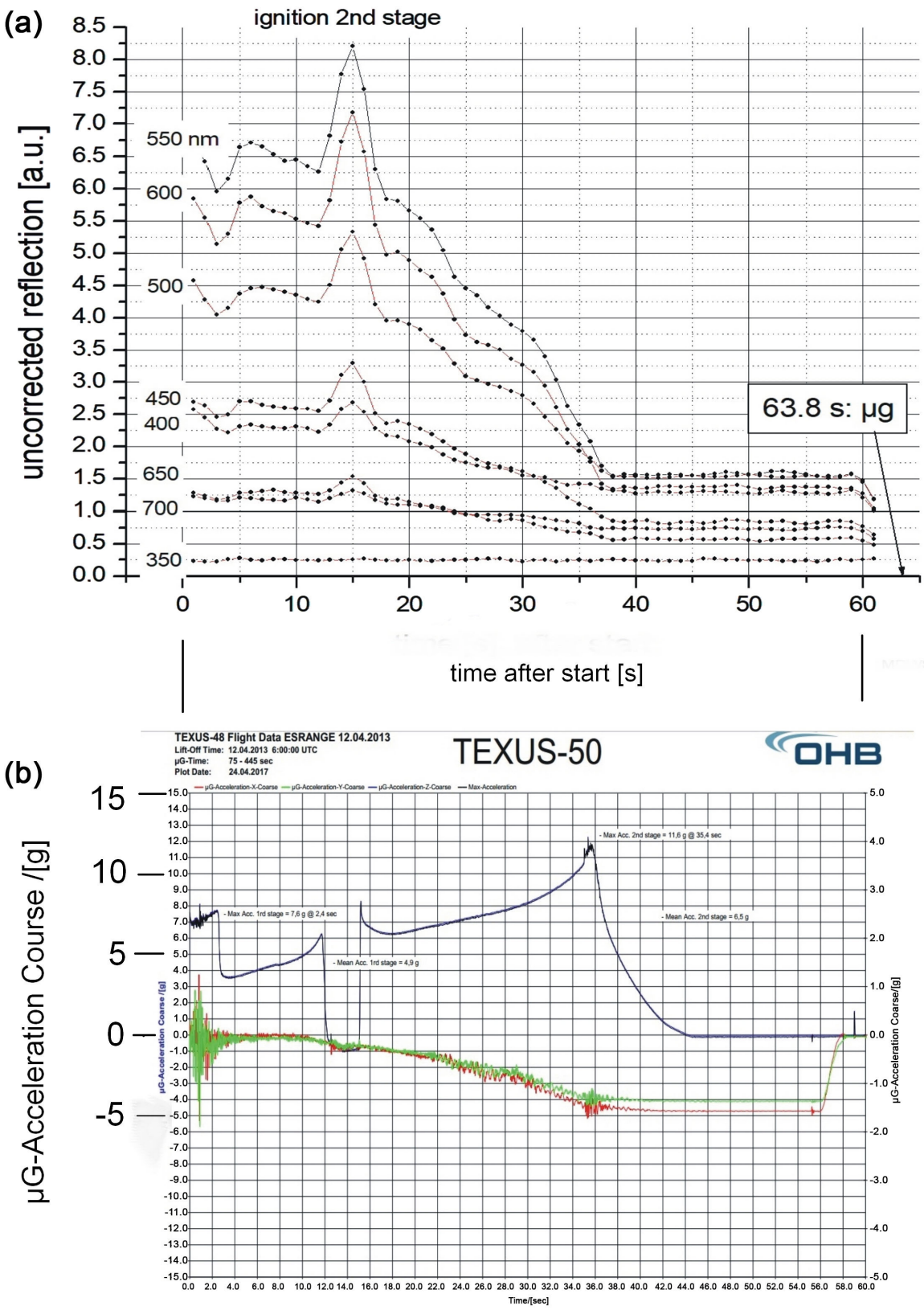

Figure 5. (a) Uncorrected reflection kinetics as measured with the diode array spectrophotometer USB-2000+ at given wavelengths extracted from data in Figure 4. Clearly, the small drop after $12 \mathrm{~s}$ is indicative of motor separation of the rockets first stage accompanied by -1 g gravity (ground situation) for $3 \mathrm{~s}$, the peak at $15 \mathrm{~s}$ represents the ignition of the second stage giving rise to a maximum of $11.6 \mathrm{~g}$. (b) Shows the acceleration time course for all three axes of the rocket, the $\mathrm{z}$-axis in flight direction and the orthogonal $\mathrm{x}$, y-axes. This diagram is the original recording supplied by Astrium. For better readability larger numbers of the G-scale are added. Interestingly, just at the maximum value of acceleration the reflection signal decreases to zero. The various offsets in the microgravity phase are caused by technical reasons and are trivial. 


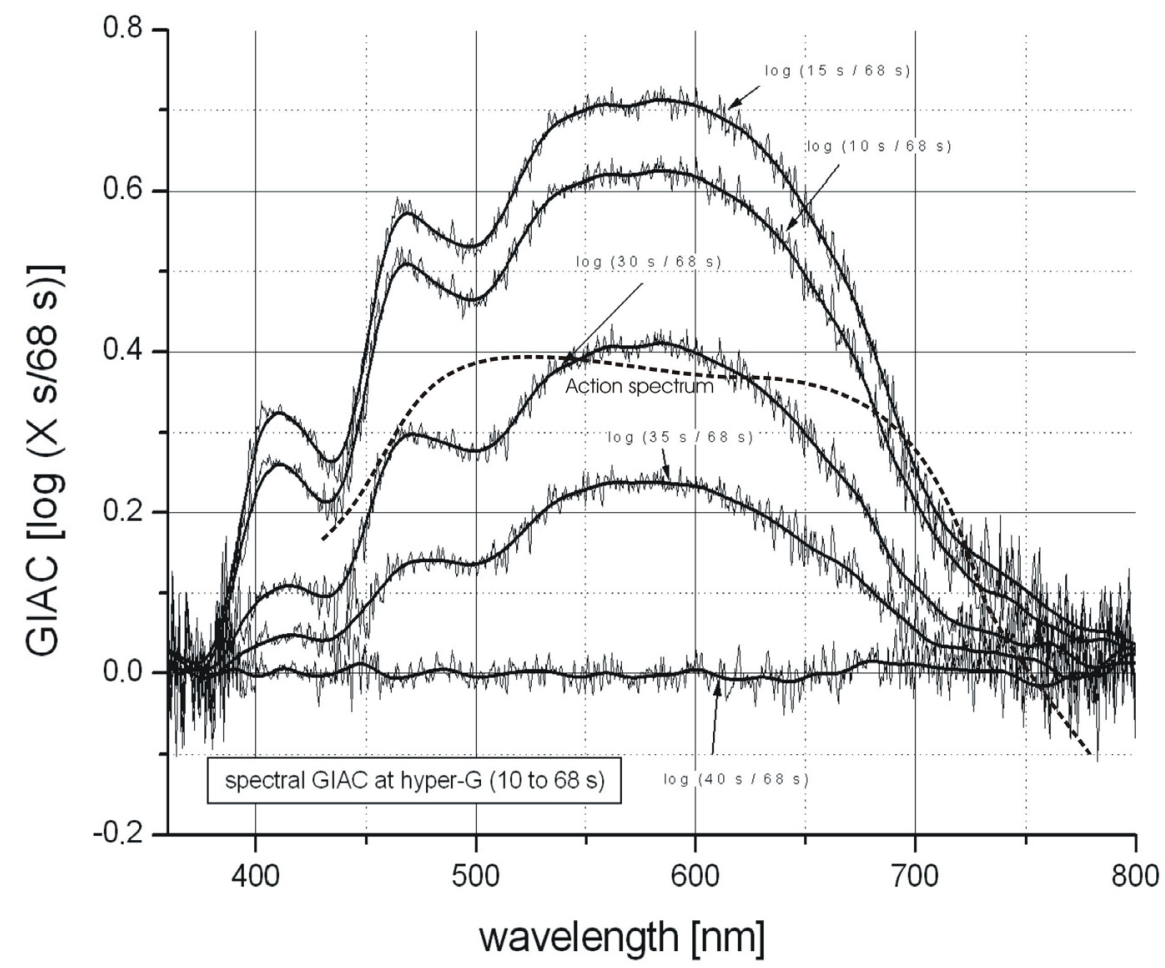

Figure 6. Shows individual corrected GIAC-spectra developing during acceleration (hypergravity, maximum $11.6 \mathrm{~g}$ ) up to microgravity as calculated from the uncorrected reflection spectra in Figure 4. A broad banded absorption spectrum comes up between 540 and above $770 \mathrm{~nm}$. Spectra are smoothed by Fourier analysis using an "inner kernel" of 50 data points. "action spectrum" (dotted line): largely averaged and smoothed GIAC spectrum obtained under moderate hypergravity conditions $(1.8 \mathrm{~g}-0 \mathrm{~g})$.

The five microgravity-spectra (Figure 7(a), Figure 7(b), extracted from Figure 4), possibly modified by gravity as generated by the centrifuge $(5,25,50$, 75 and $100 \mathrm{mg}$ ) are presented during the centrifugal ramps defined by the first paper of this series [1]. Calculating the logarithm of the five quotient spectra representing GIAC $=\Delta R=\log R_{x} / R_{0}=\Delta A=\log R_{x} / R_{0}$ at times $t_{x}$ and $t_{0}$ corresponding to the five rotational ramps with maxima at 165 (137), 221 (193), 277 (249), 333 (305) and 380 (361) s (time position of maximum at $t_{1}$, of reference spectra at $\mathrm{t}_{0}$ in brackets) do not reveal any visible difference rather than straight lines Figure $7(\mathrm{a})$. These are shown on a largely magnified scale $(\times 35,000)$, Figure 7(b). Using a Fourier smoothing function with an inner kernel corresponding to the ramp width (50 s) also does not reveal any reflection (absorption) change. Here only one difference spectrum referring to the $100 \mathrm{mg}$ ramp is shown by the white line where the MDWS-measurement reveals a clear cut signal. Concluding, in contrast to the MDWS-measurement in Paper 1 of this series [1], the SBS does not allow detecting the expected miniscule GIAC signals on a spectral basis. This reminds us of the fact that the well-studied plant photoreceptor Phytochrome also has never been measured in vivo other than by dual wavelength spectroscopy. Only after considerable accumulation the first spectral measurement of Phytochrome could be performed in vitro [6]. The (uncorrected) 
(a)

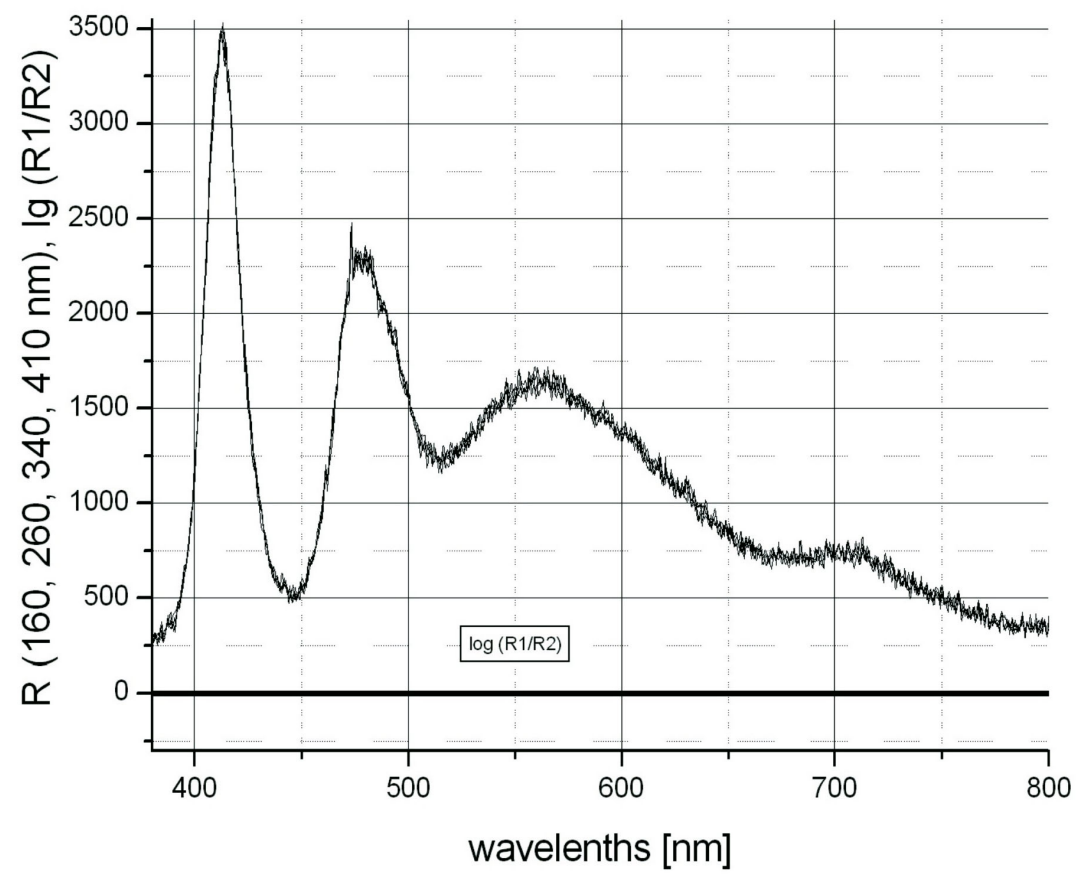

(b)

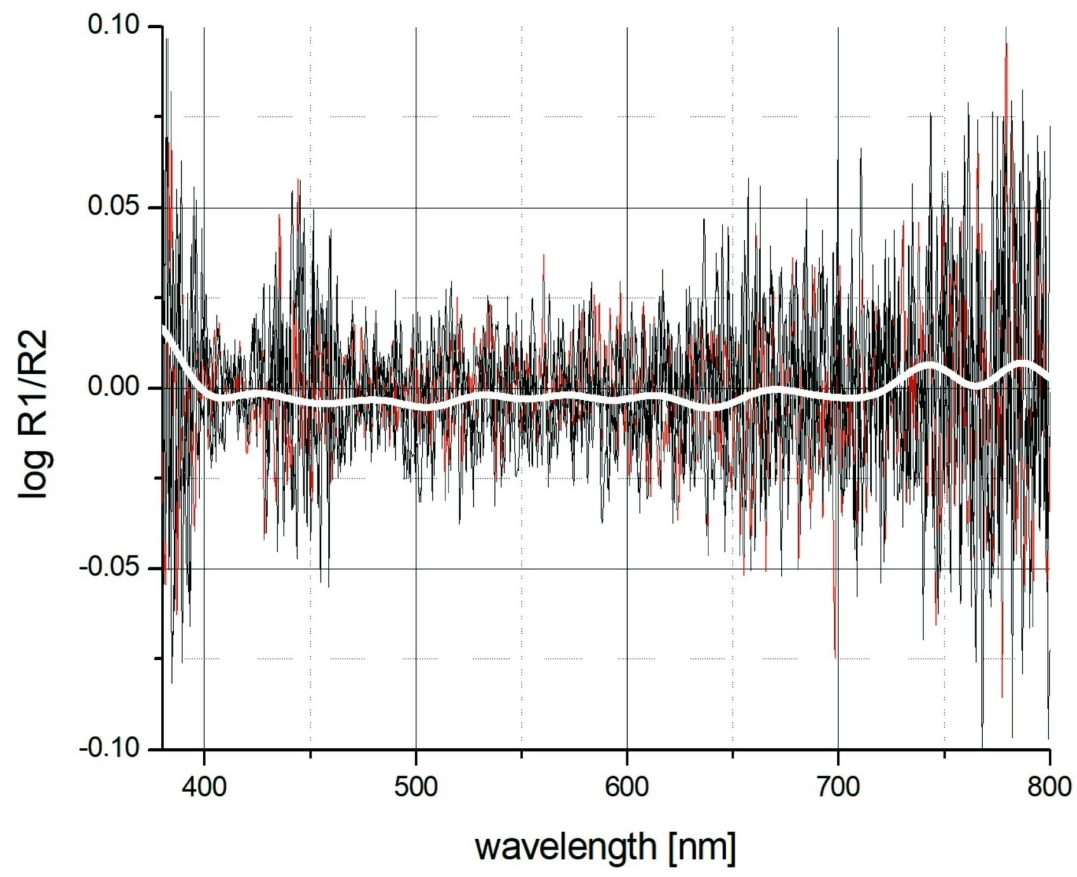

Figure 7. (a) According to MDWS-data five difference spectra are expected representing GIAC $=\Delta \mathrm{R}=\log \mathrm{R}_{1} / \mathrm{R}_{0}=\Delta \mathrm{A}=\log \mathrm{A}_{1} / \mathrm{A}_{0}$ at times $\mathrm{t}_{1}$ and $\mathrm{t}_{0}$ corresponding to the five rotational ramps. However, these plots do not reveal any difference spectra rather than straight lines at zero. (b) Shows all five difference spectra of (a) on a largely magnified scale $(\times 35,000)$.

reflection spectra of the SPPHs (Figure 4) essentially represent the spectral emission of the exciting light diodes "white + blue".

The MDWS-signal during the acceleration phase (Figure 8) is also in 


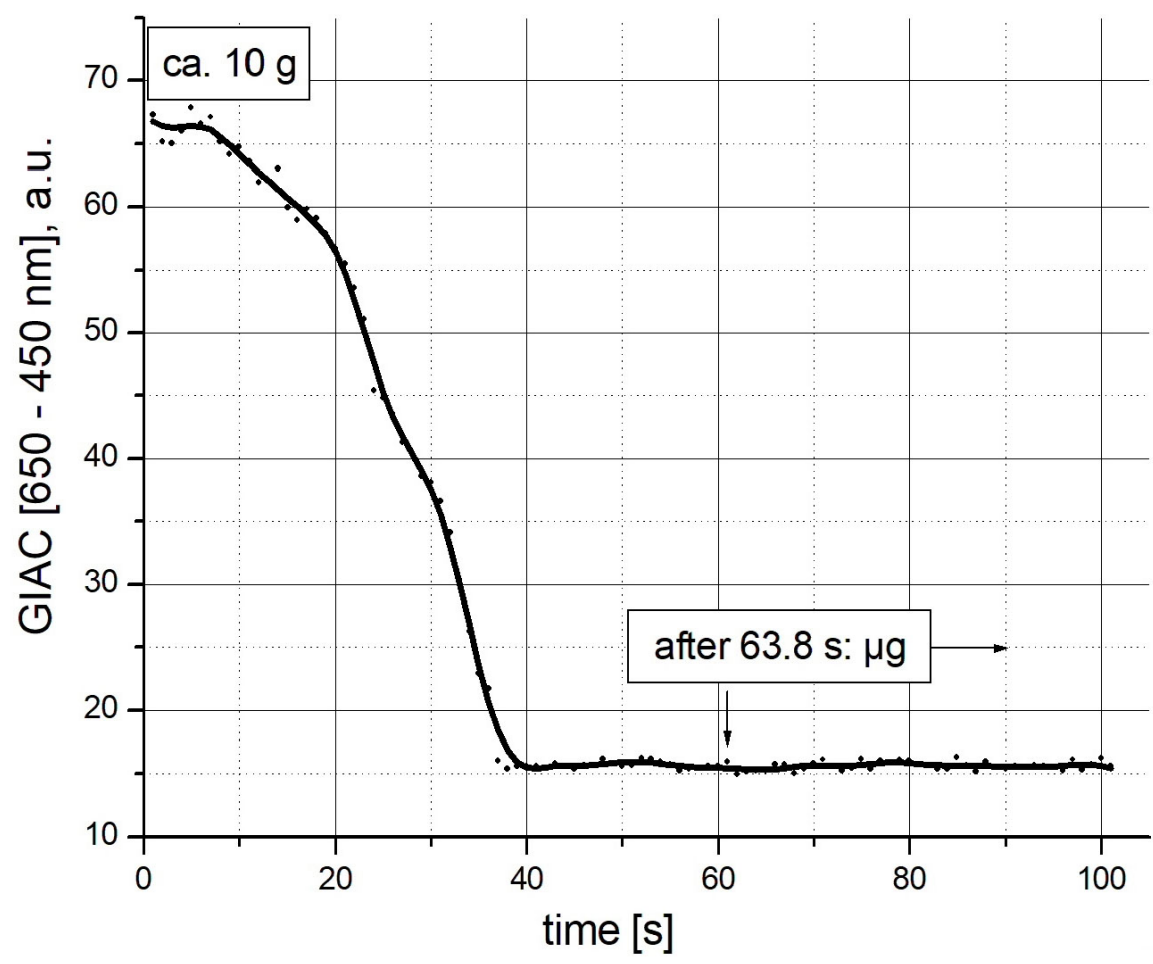

Figure 8. MDWS-signal during the acceleration phase is in consistency with non-artificial, i.e. biologically founded spectral changes seen in Figure 4. In consistency with Figure 5, the signal decreases to zero after $40 \mathrm{~s}$. The peak in Figure 5(a) after $15 \mathrm{~s}$ upon motor1-separation is not manifested in this plot.

consistency with non-artificial, i.e. biologically founded spectral changes seen in Figure 4. In consistency with Figure 5(a), the signal decreases to zero $40 \mathrm{~s}$ after liftoff (the offsets are trivial, vide supra).

Close inspection of Figure 4 reveals a smaller peak at $700 \mathrm{~nm}$ coming up at the end of the hypergravity phase. This is more clearly emphasized in Figure 9(a) showing the section of Figure 4 between 650 and $800 \mathrm{~nm}$. The final reentry of the rocket leads to extraordinary deceleration values up $37.8 \mathrm{~g}$, completely destroying Phycomyces sporangiophores excluding their spectral measurement (possibly permanent species?). Interestingly, such an absorption peak at $700 \mathrm{~nm}$ has been observed some 18 years ago in a GIAC-spectrum in a dense "hedge" of SPPHs [3], Figure 9(b). A highly interesting fact needs to be stressed: whereas under earthbound conditions $(-1 \mathrm{~g})$, this peak develops within about the first 30 min, under hypergravity conditions it comes up already after some 10 seconds. Its biochemical identification also remains unknown, so far.

A series of earth-bound test spectra of dummies made from thin copper wire (diameter $50 \mu \mathrm{m}$,) representing the Phycomyces sporangiophore (Figure 1) is shown in Figure 10(a). The constant illuminating light source is a white LED. The dummies are positioned at different distances to the spectrometer entrance (Figure 2) mimicking a presumed and "uncontrollable" mechanical movement (i.e. varying distances) of the samples in the hypergravity phase, giving rise to the GIAC-spectra seen in Figure 4. The relative light intensities range from 350 down 
to 25 arbitrary units. Figure 10(b) Clearly, the calculated reflection/absorption changes essentially show straight lines and do not cause distinctive "spectra", suggesting that the observed GIACs in Figure 6 are real and not trivial "mechanical artefacts", as could be claimed.

\section{Conclusion}

This present paper shows for the first time that hypergravity induces both

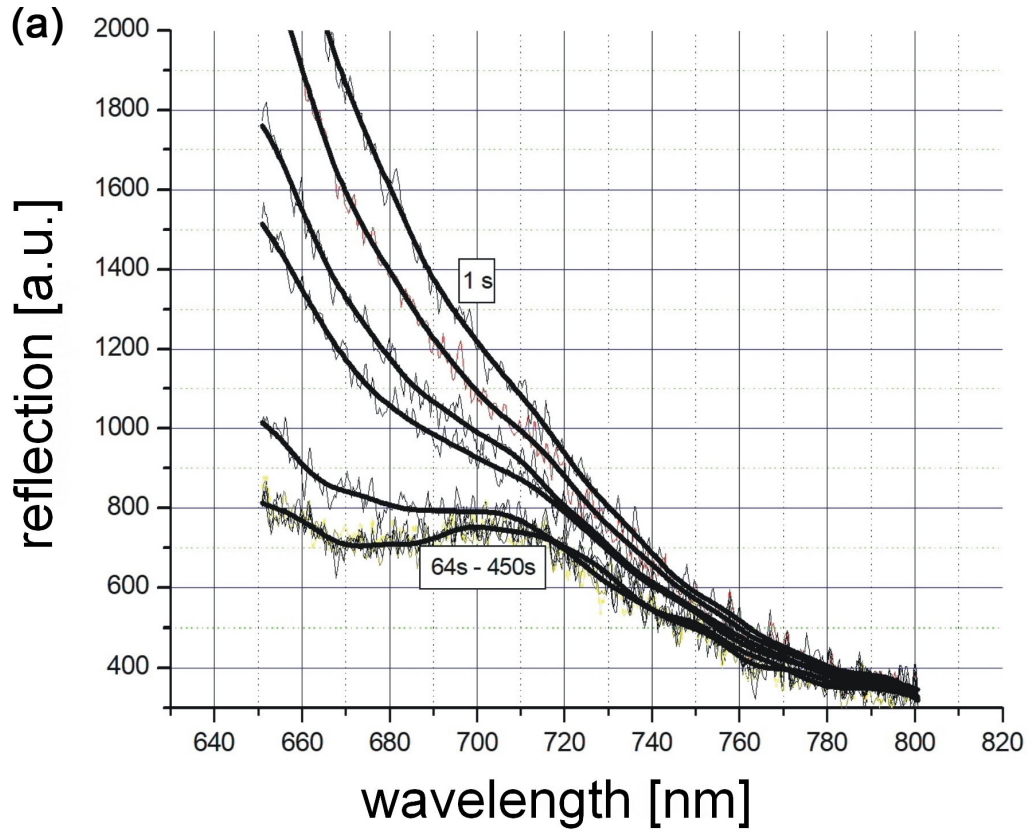

(b)

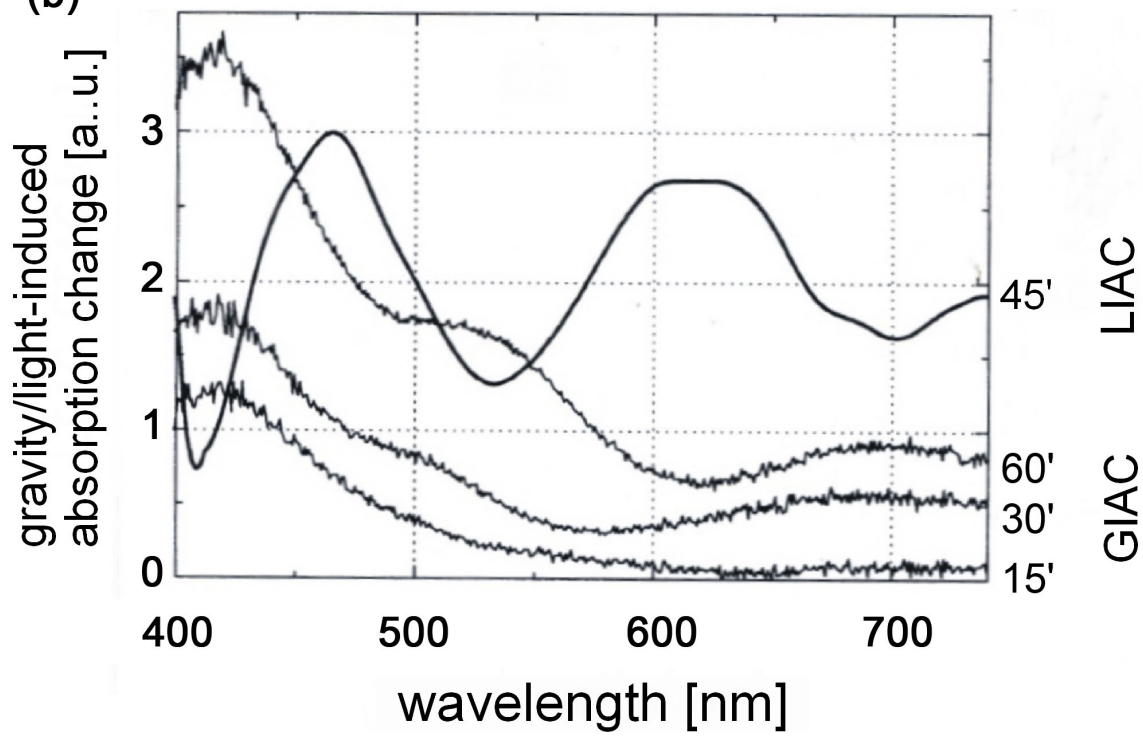

Figure 9. (a) shows the section of Figure 4 between 650 and $800 \mathrm{~nm}$. Clearly, with the decreasing GIAC-signal at decreasing hypergravity another broad peak at $700 \mathrm{~nm}$ evolves. (b) long term GIACs (after 15, 30, $60 \mathrm{~min}$ ) and LIAC (45') in SPPHs as measured some 18 years ago [8]. Clearly, under standard conditions $(1 \mathrm{~g})$ if SPPHs are tilted in the horizontal position GIACs slowly develop with characteristic broad spectral peaks at 700, 540 and $420 \mathrm{~nm}$. However, only the peak at $700 \mathrm{~nm}$ clearly appears to develop under (fast) hypergravity. 
(a)

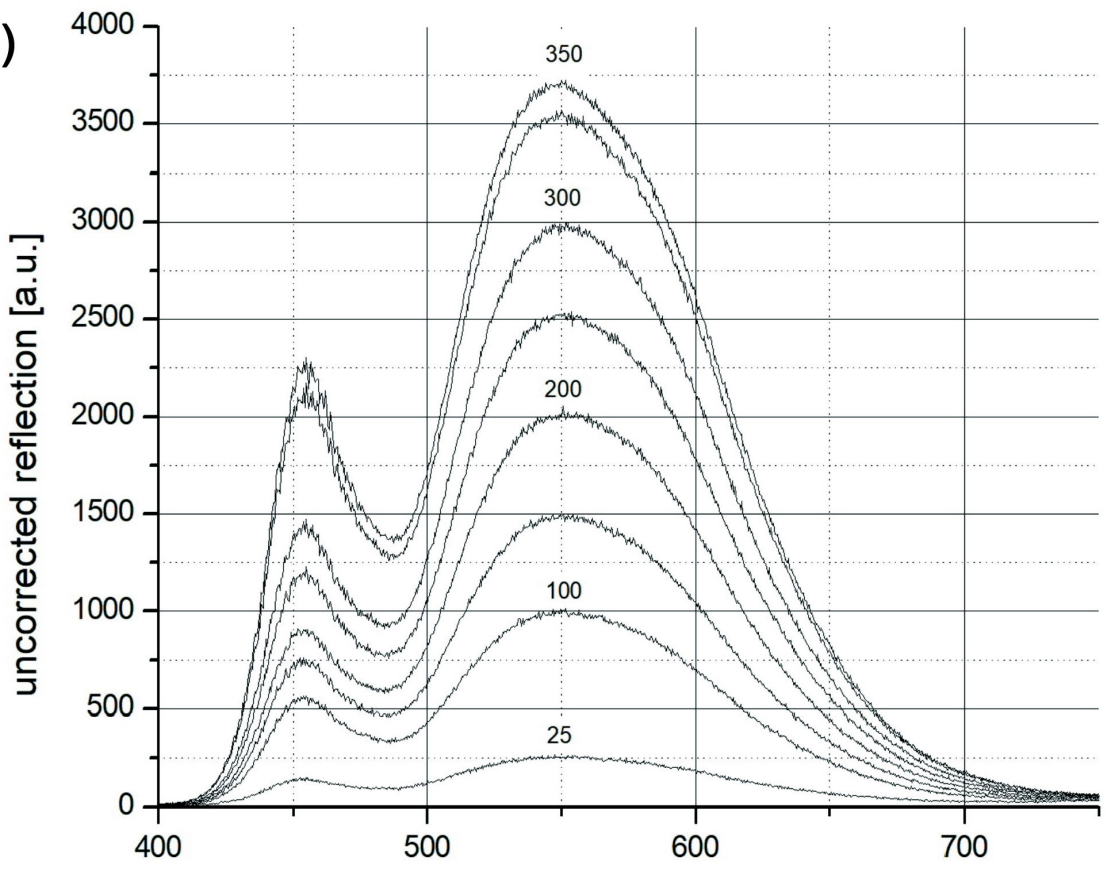

(b)

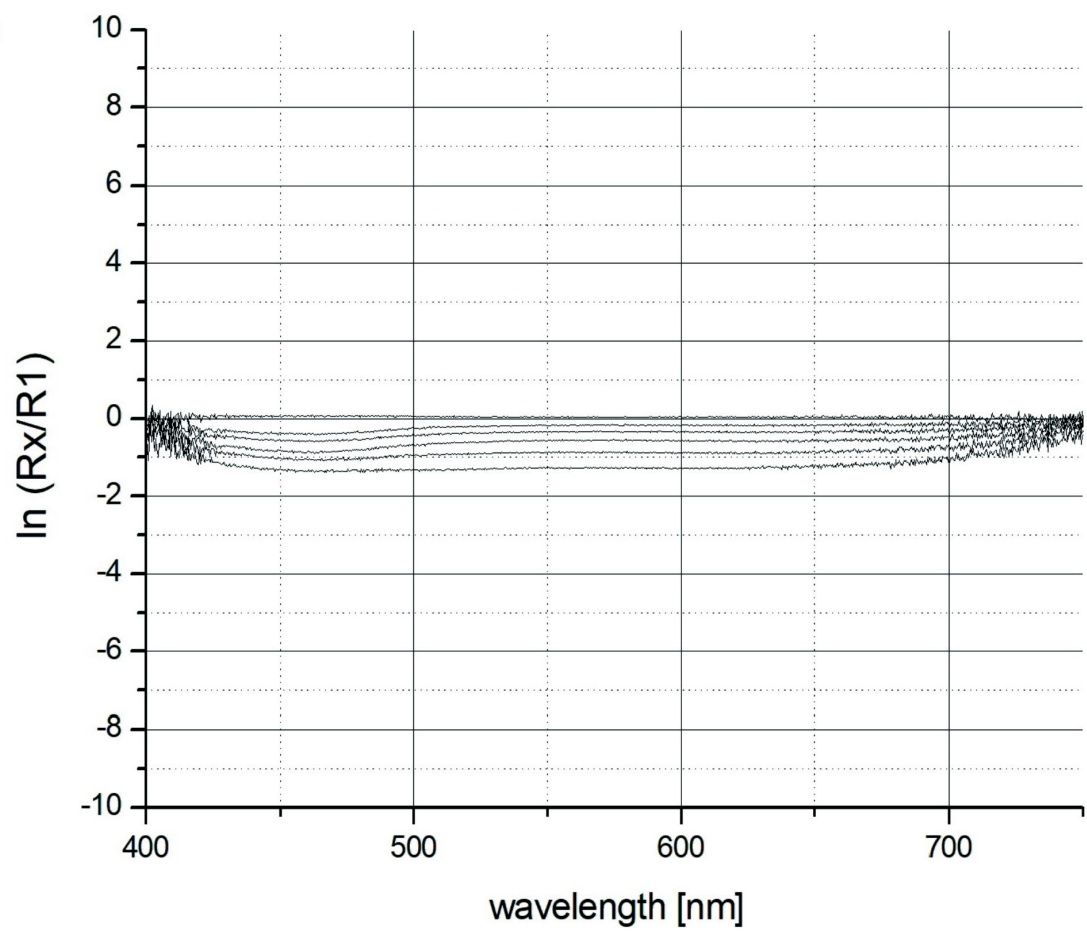

Figure 10. (a) A series of earth-bound test spectra of dummies made from thin copper wire representing the thin Phycomyces sporangiophores (Figure 1). The constant illuminating light source is a white LED. The dummies are set to different distances to the spectrometer entrance (Figure 1) mimicking a presumed and "uncontrollable" mechanical movement (i.e. varying distances) of the samples in the hypergravity phase giving rise to the GIAC-spectra seen in Figure 4.

transient as well as possibly permanent reflection/absorption changes in Phycomyces sporangiophores, which reflect molecular species which-so far-remain 
unidentified. These spectral identities generated by hypergravity within the seconds time range appear similar to those detected earlier within the minutes time range under standard 1 g-conditions (Figure 5, Figure 6, Figure 8, Figure 9). Synopsis of both papers referring to Texus 50, [1] and the present one clearly demonstrate the superior sensitivity of dual wavelength compared to single beam spectroscopy: GIACs observed under microgravity conditions by dual wavelength spectroscopy cannot be seen by common single beam spectroscopy.

\section{Acknowledgements}

The work was supported by grant 50WB1025 from the DLR/BMWI (Deutsches Zentrum für Luft- und Raumfahrt, and Bundesministerium für Wirtschaft). I thank my college Paul Galland for fruitful discussions and greatly acknowledge the excellent implementation and miniaturization of our various spectrophotometers (MDWS and USB-2000+) as formerly used for parabolic flights and now in sounding rocket campaigns by the whole team of Astrium (Bremen, Dep. TO4-BEOS, Astrium, 28199 Bremen, GmbH), particularly Thomas Hülsing. The engineers of the former Kayser-Threde GmbH (München, since $1^{\text {st }}$ Sept. 2014 they are fused with OHB Systems under the name OHB System AG, Oberpfaffenhofen, Germany) are thanked for their excellent work controlling the flight of the rocket and delivering (Figure 5(b)).

\section{References}

[1] Schmidt, W. (2017) Journal of Modern Physics, 6, 1381-1389. http://file.scirp.org/pdf/JMP_2015082810060783.pdf https://doi.org/10.4236/jmp.2015.610143

[2] Schmidt, W. (2004) Microgravity-Science and Technology, 15, 42-48. https://doi.org/10.1007/BF02870951

[3] Schmidt, W. (2004) Journal of Biochemical and Biophysical Methods, 58, 15-24. https://doi.org/10.1016/S0165-022X(03)00153-2

[4] Schmidt, W. (2005) Optical Spectroscopy in Life Sciences and Chemistry. An Introduction. Wiley-VCH, p. 369.

[5] Schmidt, W. and Galland, P. (2004) Plant Physiology, 135, 183-192. https://doi.org/10.1104/pp.103.033282

[6] Butler, W.L., Hendricks, S.B. and Siegelmann, H.W. (1964) Photochemistry and Photobiology, 3, 521-528. https://doi.org/10.1111/j.1751-1097.1964.tb08171.x

[7] Schmidt, W. (2006) Protoplasma, 229, 125-131. https://doi.org/10.1007/s00709-006-0217-8

[8] Schmidt, W. and Galland, P. (2000) Planta, 210, 848-852. https://doi.org/10.1007/s004250050689 


\section{Abbreviations}

DLR: $\quad$ Deutsches Zentrum für Luft- und Raumfahrt

ESRANGE: European Space and Sounding Rocket Range (near Kiruna, Sweden)

FFT ${ }^{2}$ : $\quad$ Fast Fourier Transform

GIAC: $\quad$ Gravity-Induced Absorption Change

MDWS: $\quad$ Micro-Dual Wavelength Spectrophotometer

RSS: $\quad$ Rapid Scan Spectrophotometer

SBS: $\quad$ Single Beam Spectrophotometer

$\mathrm{SNR}^{3}$ : $\quad$ Signal to Noise Ratio

SPPH: $\quad$ Phycomyces sporangiophore

TEXUS 50: $\quad 50^{\text {th }}$ sounding rocket campaign, name of the rocket

${ }^{2}$ There are many different FFT algorithms involving a wide range of mathematics, from simple complex-number arithmetic to group theory and number theory. Here it is used for smoothing. ${ }^{3}$ Signal-to-noise ratio (abbreviated SNR) is a measure used in science and engineering that compares the level of a desired signal to the level of background noise. 